\title{
Enterovirus D68 serosurvey: evidence for endemic circulation in the Netherlands, 2006 to 2016
}

Eveliina Karelehto ${ }^{1}$, Gerrit Koen ${ }^{1}$, Kimberley Benschop², Fiona van der Klis², Dasja Pajkrt², Katja Wolthers ${ }^{1}$

1. Department of Medical Microbiology, Laboratory of Clinical Virology, Amsterdam University Medical Center, University of Amsterdam, Amsterdam, the Netherlands

2. National Institute for Public Health and the Environment, Bilthoven, the Netherlands

3. Department of Pediatric Infectious Diseases, Emma Children's Hospital, University Medical Centers, University of Amsterdam, Amsterdam, the Netherlands

Correspondence: Katja C Wolthers (k.c.wolthers@amc.uva.nl)

Citation style for this article:

Karelehto Eveliina, Koen Gerrit, Benschop Kimberley, van der Klis Fiona, Pajkrt Dasja, Wolthers Katja. Enterovirus D68 serosurvey: evidence for endemic circulation in the Netherlands, 2006 to 2016. Euro Surveill. 2019;24(35):pii=1800671. https://doi.org/10.2807/1560-7917.ES.2019.24.35.1800671

Background: Enterovirus D68 (EV-D68) has caused major outbreaks of severe respiratory illness worldwide since 2010. Aim: Our aim was to evaluate EV-D68 circulation in the Netherlands by conducting a serosurvey of EV-D68 neutralising antibodies ( $\mathrm{nAb}$ ) among the Dutch general population. Methods: We screened 280 sera from children and adults in the Netherlands and used two independent sets of samples collected in the years 2006 and 2007 and in the years 2015 and 2016, time points before and after the first EV-D68 upsurge in 2010. Neutralisation capacity of the sera was tested against the prototype Fermon EV-D68 strain isolated in 1962 and against a recent EV-D68 strain (genotype B3) isolated in France in 2016. Results: Regardless of the time of serum collection, we found remarkably high overall seropositivity (94.3-98.3\%) for nAb against both EV-D68 strains. Geometric mean titres increased in an age-dependent manner. Conclusions: Our data suggest that EV-D68 has been circulating in the Netherlands for decades and that the enterovirus surveillance does not accurately capture the prevalence of this clinically relevant pathogen.

\section{Introduction}

Enterovirus D68 (EV-D68), belonging to the Enterovirus D species within the Picornaviridae family, was first isolated in 1962 but not frequently detected before 2010 when it started causing large outbreaks of severe respiratory illness worldwide [1-5]. Clinical symptoms commonly associated with EV-D68 infection include fever, wheezing, cough and dyspnoea [1]. Young children and individuals with underlying conditions are at high risk of developing severe lower respiratory tract disease requiring admission to an intensive care unit (ICU) and mechanical ventilation $[1,2,5]$. The characteristics of EV-D68, such as acid lability of the virions, the respiratory transmission route and symptomatology in patients, resemble those described for the related rhinoviruses [6]. However, similar to poliovirus (PV) and enterovirus $A 71$ (EV-A71), EV-D68 has the potential to spread to the central nervous system (CNS) causing neurological complications [2]. Acute flaccid myelitis (AFM) in children has been associated with EV-D68 infection [7-12].

Based on the viral capsid protein VP1 nucleotide sequence, EV-D68 isolates are classified into three clades A to C, all of which co-circulate globally [3]. In the Netherlands, EV-D68 has been detected sporadically since 1996 and the first upsurge of EV-D68 cases was reported in 2010 [4]. Continuous circulation has been observed from 2011 to 2016, with severe outbreaks in 2014 and 2016 [13-15]. Surveillance of enteroviruses (EV) occurs via the national public health networks in the context of the World Health Organization (WHO) polio surveillance, by detection of viruses from patients [16]. However, as most EV infections are asymptomatic or cause mild disease and since EV diagnostic testing is performed primarily on stool samples, detection rates are likely to account for only a minority of the true EV-D68 incidence [17].

Presence of neutralising antibodies (nAb) in serum is a widely accepted correlate of immunity and protection against severe disease associated with EV infection [18]. Thus, age-stratified serosurveys of nAb are a valuable method of understanding the prevalence of EV-D68 and evaluating the risk of an outbreak among the general population. As a part of the European NonPolio Enterovirus Network (ENPEN) [19], we aimed to characterise the seroprevalence of nAb against EV-D68 among children and adults in the Netherlands.

\section{Methods}

We screened sera collected from the population in the Netherlands before and after the 2010 EV-D68 upsurge against two strains of EV-D68: the prototype Fermon strain so that data would be comparable to previous 
FIGURE 1

Overall seroprevalence and geometric mean titre of neutralising antibodies against enterovirus D68 (prototype Fermon strain or a genotype B3 clinical isolate), the Netherlands, 2006-07 and 2015-16 $(\mathrm{n}=280)$

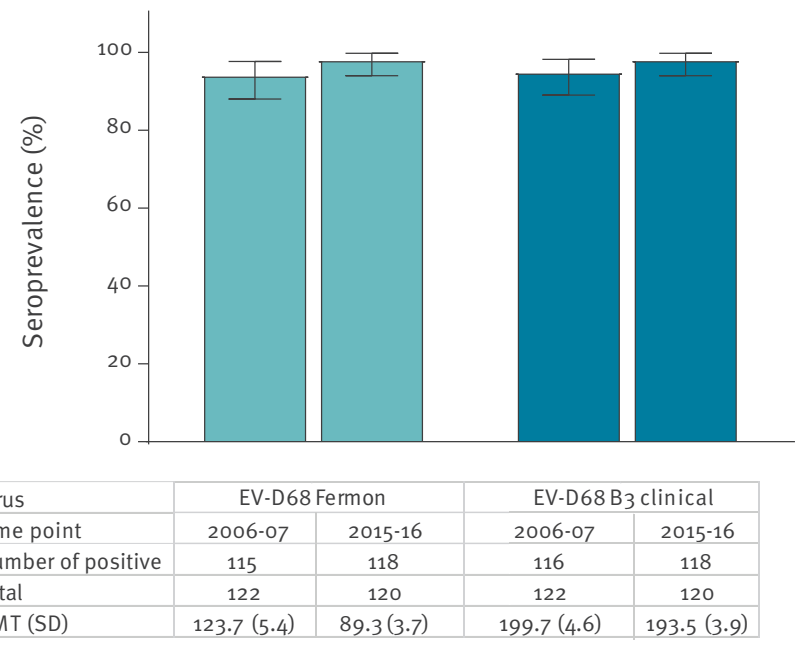

EV: enterovirus; GMT: geometric mean titre; $\mathrm{nAB}$ : neutralising antibodies; SD: standard deviation.

Light grey bars: Fermon strain; dark grey bars: B3 clinical isolate. Left columns: 2006-07; right columns: 2015-16. Whiskers indicate the $95 \%$ confidence intervals. Number of positives refers to subjects with titres $>8$.

studies done with the Fermon strain [20] and a genotype B3 clinical isolate from 2016, a contemporary circulating strain in Europe. We analysed the seropositivity and $n A b$ titre distribution in the context of time of collection, age, sex and virus strain.

\section{Enterovirus D68 viruses and cell lines}

The EV-D68 Fermon prototype strain (isolated in 1962) was obtained from the National Institute for Public Health and the Environment (RIVM, Bilthoven, the Netherlands). The EV-D68 genotype B3 clinical strain was isolated from a patient in 2016 in France and was a kind gift from Dr Bailly (Université Clermont Auvergne, Clermont-Ferrand, France). Both virus strains were cultured at $37^{\circ} \mathrm{C}, 5 \% \mathrm{CO}_{2}$ in rhabdomyosarcoma cell line (RD99; American Type Culture Collection, Manassas, United States (US)). Cells were maintained in Eagless minimum essential medium (EMEM; Lonza, Basel, Switzerland) supplemented with $8 \%$ heat-inactivated fetal bovine serum (FBS; Sigma-Aldrich, St. Louis, US), streptomycin (100 $\mathrm{\mu g} / \mathrm{mL}$; Lonza Bio Whittaker), penicillin (100 U/mL; Lonza Bio Whittaker), non-essential amino acids (NEAA; ScienCell Research Laboratories, Carlsbad, US) and L-glutamine (200 nM; Lonza, Basel, Switzerland). Chloroform treatment of the virus stocks was performed as described in the WHO Polio Manual [21]. Briefly, $10 \%(\mathrm{v} / \mathrm{v})$ chloroform (Sigma-Aldrich, St. Louis, US) was added to each virus culture and vortexed vigorously for $5 \mathrm{~min}$. Chloroform was removed by centrifugation for $10 \mathrm{~min}$ at $3000 \mathrm{rpm}$. The $50 \%$ tissue culture infective dose (TCID50) of virus stocks was determined by means of end-point dilution using the Reed and Muench method [22].

\section{Serum samples}

We screened 280 anonymised serum samples from Dutch individuals aged $0-79$ years. We used two independent sets of samples collected at time points before and after the 2010 EV-D68 upsurge in the Netherlands. Sera from 2006 and 2007 were obtained from the RIVM as part of the PIENTER2 study (Dutch acronym for the survey on the immunisation effect in the Netherlands for evaluation of the national immunisation programme: Peiling Immunisatie Effect Nederland Ter Evaluatie van het Rijksvaccinatieprogramma [23]).

\section{Neutralisation assay}

The sera were tested using a previously described neutralisation assay [24]. Heat-inactivated sera were serially diluted in 96-well microtitre plates in a volume of $50 \mu \mathrm{L}$ per well and incubated with 100 TCID50 per 50 $\mu \mathrm{L}$ per well of EV-D68. Subsequently, $100 \mu \mathrm{L}$ of RD99 cells were added and incubated for 7 days. Neutralising titres were calculated based on cytopathogenic effect using the Reed and Muench method and reported as the reciprocal titres of serum dilutions exhibiting $50 \%$ neutralisation [22]. An $n A b$ titre of $\geq 1: 8$ was considered positive. In agreement with previous publications [20], we defined titres 8-64 as 'low', 64-128 as moderate, 128-512 as 'high' and >512 as 'very high'.

\section{Statistical analysis}

Data were grouped in categories based on the following: the EV-D68 virus strain used in the assay (prototype Fermon or genotype B3 clinical isolate), serum collection time point (2006-07 or 2015-16), serum donor sex (male or female) and serum donor age. The overall EV-D68 nAb seroprevalences between different groups were compared using chi-squared tests. Kruskal-Wallis test with Dunn's post hoc analysis was used to compare the overall and the age-stratified geometric mean titres between the prototype Fermon strain and the genotype $B_{3}$ clinical isolate. One-way ANOVA with Tukey's multiple comparisons test was used to compare the geometric mean titres between the age groups. Childrenyounger than 1 year were excluded from the overall seroprevalence and overall geometric mean titre analyses because of the potential presence of maternal antibodies against EV-D68. Data were analysed using IBM SPSS Statistics for Windows, Version 23.0 (IBM Corp., Armonk, US) and GraphPad Prism 7 (GraphPad Software Inc., La Jolla, US) with a significance level of $p<0.05$.

\section{Ethical statement}

The sera had been collected by population-based sampling approved by the Medical Ethics Testing Committee of the Foundation of Therapeutic Evaluation of Medicines (ISRCTN 20164309) [25]. Sera collected between 2015 and 2016 were residual samples from hospitalised patients and staff at the University Medical Centers (Amsterdam, the Netherlands). No 
Age-stratified seroprevalence and distribution of enterovirus D68 neutralising antibodies against the prototype Fermon strain or the genotype B3 clinical isolate, the Netherlands, 2006-07 and 2015-16 ( $\mathrm{n}=280)$

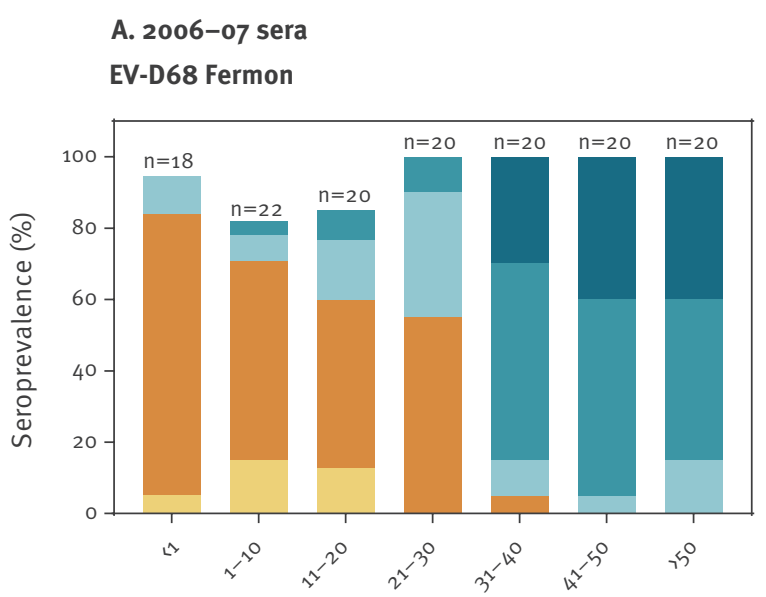

Age group (years)

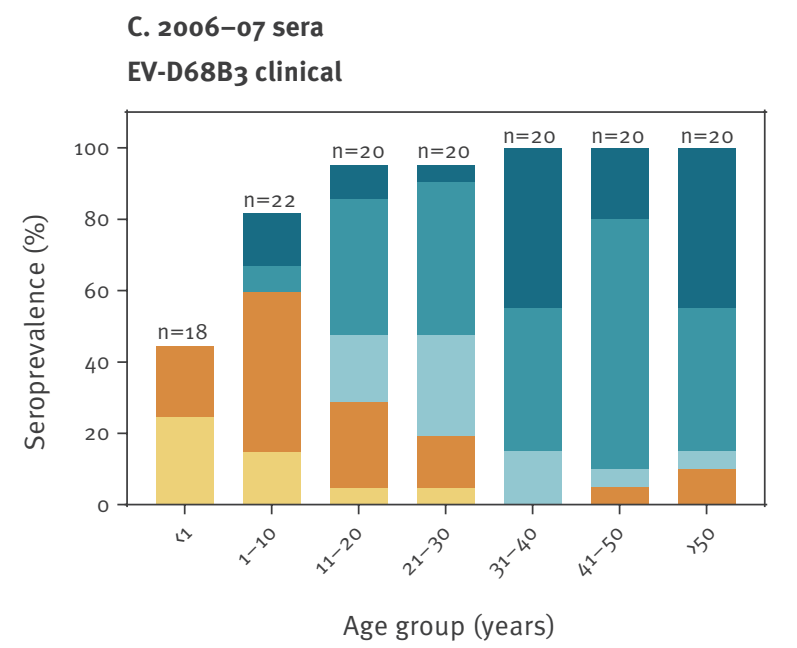

EV: enterovirus; $n A B$ : neutralising antibodies.

ethical approval is required for anonymous use of residual serum in the Netherlands.

\section{Results}

High overall seropositivity for enterovirus D68 neutralising antibodies

As depicted in Figure 1, the overall EV-D68 nAb seroprevalence rates and geometric mean titres (GMT) were high, with no statistically significant differences between time points before and after the 2010 EV-D68 upsurge in the Netherlands or between the virus strains. The overall nAb seroprevalence in the 2006 and 2007 sera against the prototype Fermon strain was $94.3 \%$ (95\% confidence interval (CI): 88.0-97.7) with a GMT of 123.7 (standard deviation (SD): 5.4) and in the 2015 and 2016 sera, it was $98.3 \%$ (95\% Cl: 94.0-99.8) with a GMT of 89.3 (SD: 3.7 ). The overall nAb seroprevalence in the 2006 and 2007 sera against the genotype
B. 2015-16 sera

\section{EV-D68 Fermon}

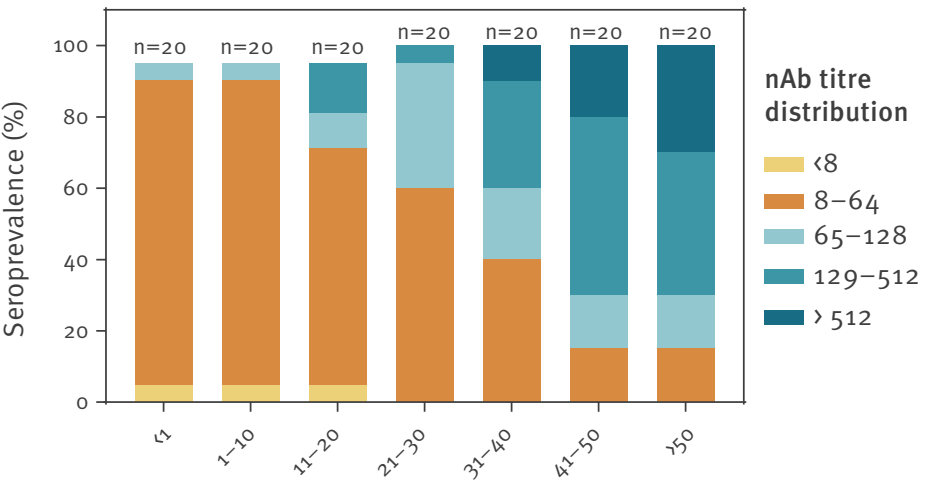

Age group (years)

\section{2015-16 sera}

EV-D68B3 clinical

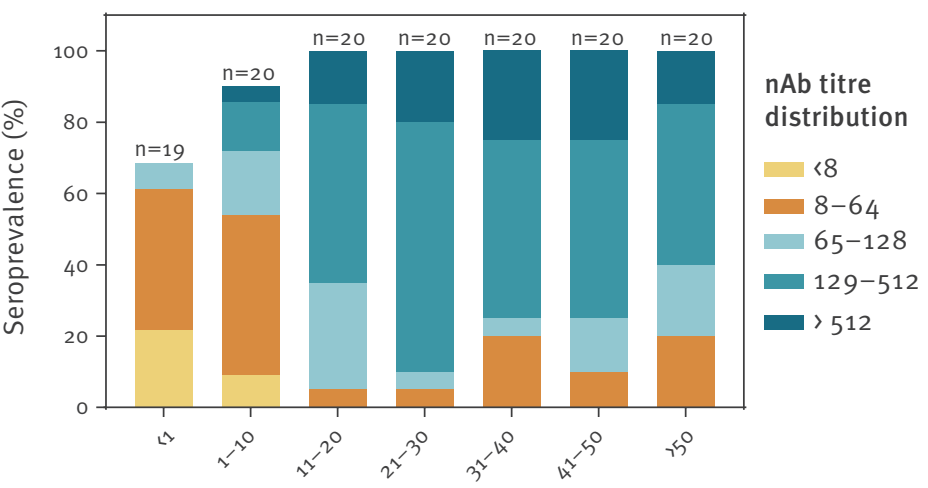

Age group (years)
B3 clinical isolate was $95.1 \%$ ( $95 \% \mathrm{Cl}: 89.0-98.2)$ with a GMT of 199.7 (SD: 4.6) and in the 2015 and 2016 sera, it was $98.3 \%$ ( $95 \% \mathrm{Cl}: 94.0-99.8$ ) with a GMT of 193.5 (SD: 3.9). No differences in the $\mathrm{nAb}$ seropositivity rates were found between female and male cases (data not shown).

\section{Age-associated increase in enterovirus D68 neutralising antibody titres}

Age-stratified analysis showed that in children below the age of 1 year the EV-D68 nAb seroprevalence was 94.4-95.0\% against the Fermon EV-D68 strain and 44.4-68.4\% against the genotype $B_{3}$ clinical isolate (Figure 2). While more infants were seropositive for $\mathrm{nAb}$ against the Fermon strain than against the genotype B3 clinical isolate, their GMT values were similar against both strains (Table). Regardless of the virus strain, seroprevalence of EV-D68 nAb was $81.8-95.0 \%$ in 1-10 year-old children, while sera from adolescents 
Geometric mean titres of neutralising antibodies against enterovirus D68, serosurvey, the Netherlands, 2006-07 and 2015-16 $(\mathrm{n}=280)$

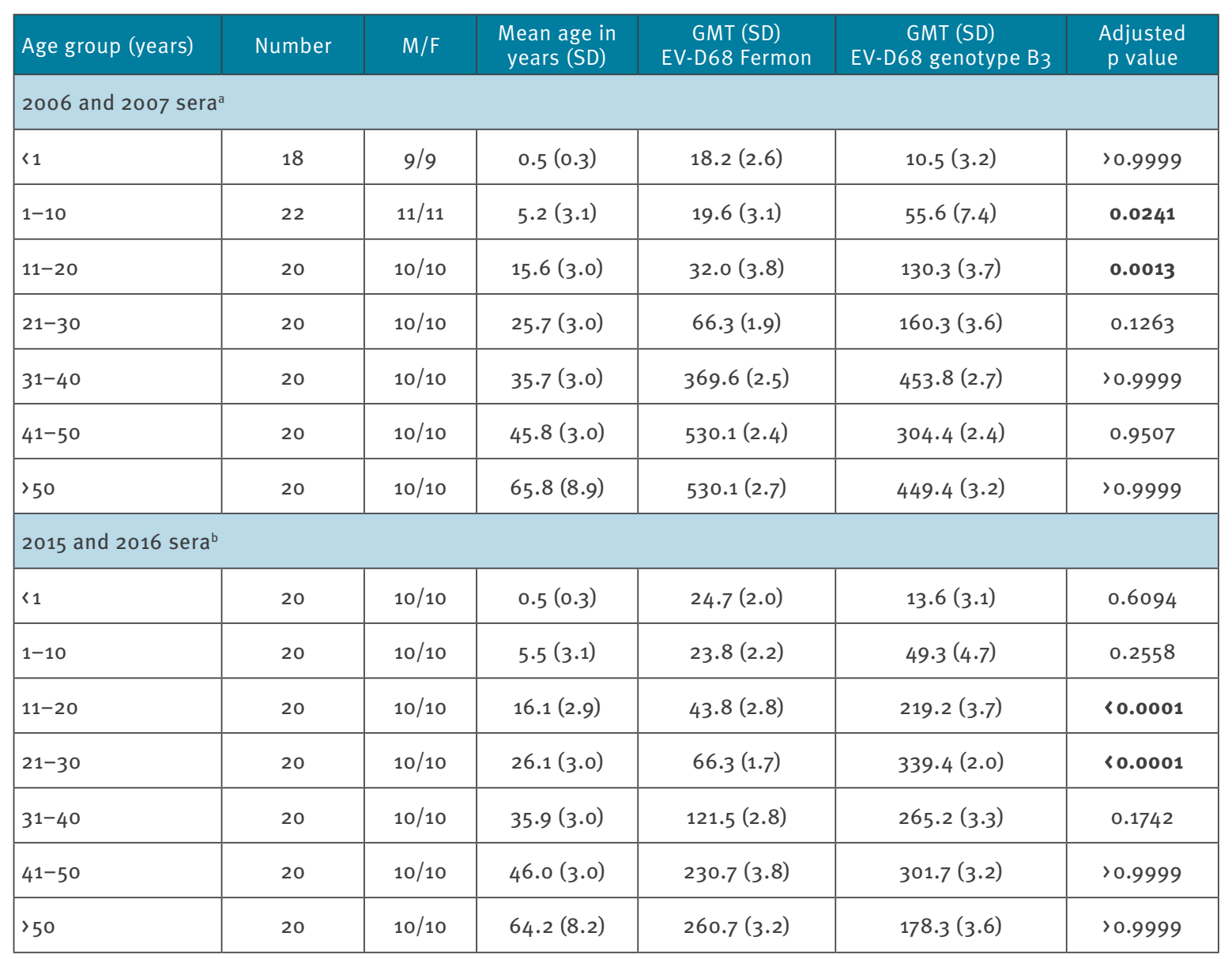

EV: enterovirus; GMT: geometric mean titre; M/F: male/female; SD: standard deviation.

a Population-based sampling, National Institute for Public Health and the Environment (RIVM), Bilthoven.

${ }^{b}$ Residual sera from hospitalised patients and staff, Academic Medical Center, Amsterdam.

Numbers in bold indicate statistical significance.

and adults (age groups 11-20, 21-30, 31-40, 41-50 and above 50 years) were $85-100 \%$ positive for EV-D68 nAb (Figure 2). Children and young adults in age groups $1-10$ and $11-20$ years had significantly lower GMT of $n A b$ against the Fermon strain than against the genotype B3 clinical EV-D68 isolate (Table). Most adult age groups had high GMTs against both virus strains with no statistically significant differences between the virus strains (Table). Statistical pairwise GMT comparisons between age groups indicated that children younger than 1 year and children between 1 and 10 years of age had significantly lower GMT of nAb against both the Fermon and the genotype $B_{3}$ clinical isolate when compared with the GMTs in the adult age groups (adjusted $p$ values $<0.0001$; Supplementary Tables $\mathrm{S}_{1}$ and $\mathrm{S}_{2}$ ).
Enterovirus D68 clinical surveillance in the Netherlands, 1996-2017

We extracted the EV-D68 case numbers reported during 1996 to 2017 in the Netherlands from the national Clinical Enterovirus Surveillance (CEVS) database (Figure 3) [26]. From 1996 to 2010, enterovirus testing was performed primarily on stool samples and few cases were observed. Because the 2010 EV-D68 outbreak was discovered via primary care surveillance done by Nivel, the Dutch Institute for Health Care Research, in respiratory samples that were not included in the CEVS, this outbreak is not visible in Figure 3 [4]. EV-D68 testing in respiratory samples has been gradually implemented following the 2010 outbreak. After 2010, 146 cases have been confirmed, most of them during an outbreak in 2016 [13].

\section{Discussion}

EV-D68 was only sporadically detected before 2010 when it suddenly caused large outbreaks of severe 
Clinical enterovirus surveillance data on enterovirus D68, the Netherlands, 1996-2017 $(\mathrm{n}=151)$

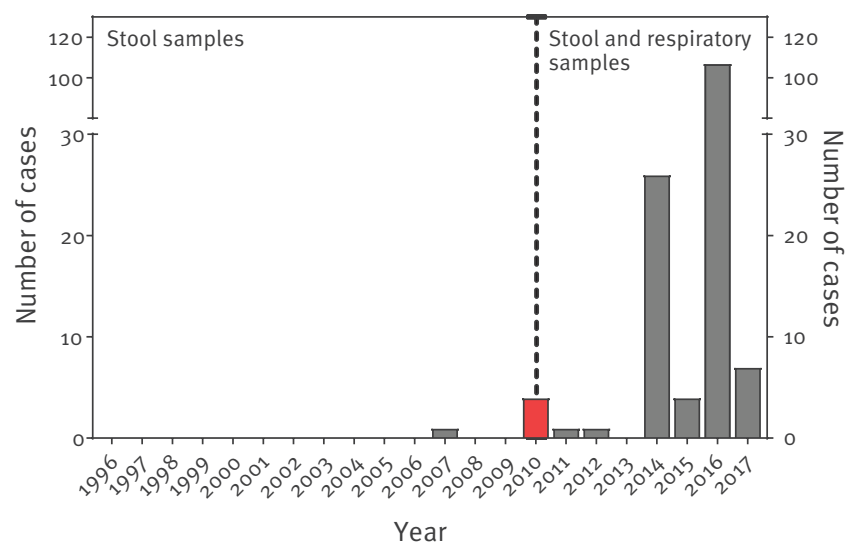

From 1996 to 2010 , enterovirus testing was performed primarily on stool samples, showing low numbers of cases in 2010, the first outbreak year in the Netherlands (highlighted in red). Since 2010 , also respiratory samples have been increasingly tested for EV-D68.

lower respiratory infections and polio-like illness worldwide, but particularly in North America $[1,4,5,9]$. Concerns were raised that EV-D68 was developing from an infrequent cause of mild disease to a major human pathogen with neurovirulent properties [1]. This study is the first serological investigation into the prevalence of EV-D68 among the Dutch population.

In line with previous sero-epidemiological studies from Finland and China, with seroprevalence rates from 90 to $100 \%[20,27]$, the overall nAb prevalence was remarkably high in sera collected both before and after the first reported EV-D68 upsurge in the Netherlands in 2010. The $n A b$ were specific to both the prototype Fermon EV-D68 strain and a recent genotype $B_{3}$ clinical isolate from France. Age-stratified analyses indicated that the overall EV-D68 nAb seroprevalence was approaching $90 \%$ or more already in 1-10 year-old children. The higher GMT in the older age groups is most likely explained by frequent boostering. Our data suggest that EV-D68 circulation has been endemic in the Netherlands for decades.

Antigenic drift has been proposed as a mechanism to explain the sudden EV-D68 emergence [4,28,29]. We found that Dutch children and young adults had higher $n A b$ titres against the recent genotype $B_{3}$ clinical isolate EV-D68 isolate than against the prototype strain. However, overall the sera from all time points and age groups could efficiently neutralise both EV-D68 strains with minimal differences between GMT. As we used anonymous serum collections, we were unable to relate the exposure histories of EV-D68 sample donors to our seroprevalence data. This is a limitation of our study. Cross-neutralisation by $\mathrm{nAb}$ elicited against other prevalent enteroviruses may be a confounding factor in our study. However, evidence of cross-neutralisation among different enterovirus serotypes is scarce $[18,30]$. Previously it was reported that EV-D68 could not be neutralised with the reference EV-D70 antiserum [29]. In the same report, it was suggested that a small antigenic variation between the 2014 outbreak viruses and the Fermon strain could explain differences in neutralisation titres.

We hypothesise that EV-D68 incidence in the Netherlands is underestimated based on the following: (i) in general, the majority of enterovirus infections are not reported as most infections are subclinical or cause only mild illness in healthy individuals [18]; (ii) standard molecular diagnostics cannot distinguish between rhinovirus and EV infection, and EV type-specific testing is predominantly based on stool sampling since EV are not perceived as relevant respiratory pathogens [17]; (iii) as reported previously in other countries $[20,27]$, we observed a nearly universal prevalence of EV-D68 neutralising antibodies among the Dutch general population.

\section{Conclusion}

We report a high level of population immunity against EV-D68 and conclude that EV-D68 has been endemically circulating in the Netherlands for decades. Our results suggest that the current EV surveillance does not accurately capture the EV-D68 prevalence in the Netherlands. In order to fully understand the EV-D68 disease burden, we propose monitoring and routine EV-D68 testing of nasopharyngeal aspirate or throat swab specimens for patients with acute respiratory presentations. Further research on antigenic variation and pathogenicity of the emerging EV-D68 variants is necessary to elucidate the factors underlying disease severity and outbreak dynamics.

\section{Acknowledgments}

Funding: This work was supported by the European Union's Seventh Framework Marie-Curie IAPP AIROPico [REA-grantagreement no 612308].

\section{Conflict of interest}

None declared.

\section{Authors' contributions}

Set-up of the study: EK, KW, DP, KB; executing the experiments: EK, GK; samples and database data deliverance: KB, FvdK; writing of the manuscript: EK, KB, DP, KW; supervision of the project: DP, KW.

\section{References}

1. Holm-Hansen CC, Midgley SE, Fischer TK. Global emergence of enterovirus D68: a systematic review. Lancet Infect Dis. 2016;16(5):e64-75. https://doi.org/10.1016/S14733099(15)00543-5 PMID: 26929196

2. Messacar K, Abzug MJ, Dominguez SR. The emergence of enterovirus-D68. Microbiol Spectr. 2016;4(3). PMID: 27337448 
3. Tokarz R, Firth C, Madhi SA, Howie SR, Wu W, Sall AA, et al. Worldwide emergence of multiple clades of enterovirus 68. J Gen Virol. 2012;93(Pt 9):1952-8. https://doi.org/10.1099/ vir.0.043935-0 PMID: 22694903

4. Meijer A, van der Sanden S, Snijders BE, Jaramillo-Gutierrez $G$, Bont L, van der Ent CK, et al. Emergence and epidemic occurrence of enterovirus 68 respiratory infections in The Netherlands in 2010. Virology. 2012;423(1):49-57. https://doi. org/10.1016/j.virol.2011.11.021 PMID: 22177700

5. Rahamat-Langendoen J, Riezebos-Brilman A, Borger R, van der Heide R, Brandenburg A, Schölvinck E, et al. Upsurge of human enterovirus 68 infections in patients with severe respiratory tract infections. J Clin Virol. 2011;52(2):103-6. https://doi. org/10.1016/j.jcv.2011.06.019 PMID: 21802981

6. Oberste MS, Maher K, Schnurr D, Flemister MR, Lovchik JC, Peters $\mathrm{H}$, et al. Enterovirus 68 is associated with respiratory illness and shares biological features with both the enteroviruses and the rhinoviruses. J Gen Virol. 2004;85(Pt 9):2577-84. https://doi.org/10.1099/vir.0.79925-0 PMID: 15302951

7. Sejvar JJ, Lopez AS, Cortese MM, Leshem E, Pastula DM, Miller L, et al. Acute flaccid myelitis in the United States, AugustDecember 2014: results of nationwide surveillance. Clin Infect Dis. 2016;63(6):737-45. https://doi.org/10.1093/cid/ciw372 PMID: 27318332

8. Aliabadi N, Messacar K, Pastula DM, Robinson CC, Leshem $E$, Sejvar JJ, et al. Enterovirus D68 infection in children with acute flaccid myelitis, Colorado, USA, 2014. Emerg Infect Dis. 2016;22(8):1387-94. https://doi.org/10.3201/eid2208.151949 PMID: 27434186

9. Midgley CM, Jackson MA, Selvarangan R, Turabelidze G, Obringer E, Johnson D, et al. Severe respiratory illness associated with enterovirus D68 - Missouri and Illinois, 2014. MMWR Morb Mortal Wkly Rep. 2014;63(36):798-9. PMID: 25211545

10. Dyda A, Stelzer-Braid S, Adam D, Chughtai AA, Maclntyre CR. The association between acute flaccid myelitis (AFM) and Enterovirus D68 (EV-D68) - what is the evidence for causation? Euro Surveill. 2018;23(3):17-00310. https:// doi.org/10.2807/1560-7917.ES.2018.23.3.17-00310 PMID: 29386095

11. Cabrerizo M, García-Iñiguez JP, Munell F, Amado A, MadurgaRevilla P, Rodrigo C, et al. First cases of severe flaccid paralysis associated with enterovirus D68 infection in Spain, 2015-2016. Pediatr Infect Dis J. 2017;36(12):1214-6. https://doi. org/10.1097/INF.0000000000001668 PMID: 28661963

12. Pfeiffer HC, Bragstad K, Skram MK, Dahl H, Knudsen PK, Chawla MS, et al. Two cases of acute severe flaccid myelitis associated with enterovirus D68 infection in children, Norway, autumn 2014. Euro Surveill. 2015;20(10):21062. https://doi. org/10.2807/1560-7917.ES2015.20.10.21062 PMID: 25788251

13. Knoester M, Schölvinck EH, Poelman R, Smit S, Vermont CL, Niesters HG, et al. Upsurge of Enterovirus D68, the Netherlands, 2016. Emerg Infect Dis. 2017;23(1):140-3. https:// doi.org/10.3201/eid2301.161313 PMID: 27660916

14. Meijer A, Benschop KS, Donker GA, van der Avoort HG. Continued seasonal circulation of enterovirus D68 in the Netherlands, 2011-2014. Euro Surveill. 2014;19(42):20935. https://doi.org/10.2807/1560-7917.ES2014.19.42.20935 PMID: 25358039

15. Poelman R, Schölvinck EH, Borger R, Niesters HG, van Leer-Buter C. The emergence of enterovirus D68 in a Dutch University Medical Center and the necessity for routinely screening for respiratory viruses. J Clin Virol. 2015;62:1-5. https://doi.org/10.1016/j.jcv.2014.11.011 PMID: 25542461

16. Harvala H, Jasir A, Penttinen P, Pastore Celentano L, Greco D, Broberg E. Surveillance and laboratory detection for non-polio enteroviruses in the European Union/European Economic Area, 2016. Euro Surveill. 2017;22(45):16-00807. https:// doi.org/10.2807/1560-7917.ES.2017.22.45.16-00807 PMID: 29162204

17. McAllister SC, Schleiss MR, Arbefeville S, Steiner ME, Hanson RS, Pollock C, et al. Epidemic 2014 enterovirus D68 crossreacts with human rhinovirus on a respiratory molecular diagnostic platform. PLoS One. 2015;10(3):eo118529. https:// doi.org/10.1371/journal.pone.0118529 PMID: 25799541

18. Ehrenfeld E, Domingo E, Roos PR. The Picornaviruses. Washington, DC: ASM Press; 2010.

19. Harvala H, Broberg E, Benschop K, Berginc N, Ladhani S, Susi P, et al. Recommendations for enterovirus diagnostics and characterisation within and beyond Europe. J Clin Virol. 2018;101:11-7. https://doi.org/10.1016/j.jcv.2018.01.008 PMID: 29414181

20. Xiang Z, Li L, Ren L, Guo L, Xie Z, Liu C, et al. Seroepidemiology of enterovirus D68 infection in China. Emerg Microbes Infect. 2017;6(5):e32. https://doi.org/10.1038/emi.2017.14 PMID: 28487560

21. World Health Organization (WHO). Polio laboratory manual 2004. 4th edition. Geneva: WHO; 2004. Available from: https://apps.who.int/iris/bitstream/handle/10665/68762/ WHO_IVB_04.10.pdf?sequence=1\&isAllowed=y

22. Reed LJ, Muench H. A simple method of estimating fifty percent endpoints. Am J Hyg. 1938;27:493-7.

23. Mollema L, de Melker HE, Hahné SJM, van Weert JWM, Berbers GAM, van der Klis FRM. PIENTER 2-project: second research project on the protection against infectious diseases offered by the national immunization programme in the Netherlands. Bilthoven: National Institute for Public Health and the Environment; 2010. Available from: https://www.rivm.nl/ publicaties/pienter-2-project-second-research-project-onprotection-against-infectious-diseases

24. Karelehto E, van der Sanden S, Geraets JA, Domanska A, van der Linden L, Hoogendoorn D, et al. Strain-dependent neutralization reveals antigenic variation of human parechovirus 3. Sci Rep. 2017;7(1):12075. https://doi. org/10.1038/S41598-017-12458-5 PMID: 28935894

25. van der Klis FR, Mollema L, Berbers GA, de Melker HE, Coutinho RA. Second national serum bank for populationbased seroprevalence studies in the Netherlands. Neth J Med. 2009;67(7):301-8. PMID: 19687529

26. Benschop KS, Rahamat-Langendoen JC, van der Avoort HG, Claas EC, Pas SD, Schuurman R, et al. VIRO-TypeNed, systematic molecular surveillance of enteroviruses in the Netherlands between 2010 and 2014. Euro Surveill. 2016;21(39):30352. https://doi.org/10.2807/1560-7917. ES.2016.21.39.30352 PMID: 27719752

27. Smura T, Ylipaasto P, Klemola P, Kaijalainen S, Kyllönen L, Sordi V, et al. Cellular tropism of human enterovirus D species serotypes EV-94, EV-70, and EV-68 in vitro: implications for pathogenesis. J Med Virol. 2010;82(11):1940-9. https://doi. org/10.1002/jmv.21894 PMID: 20872722

28. Imamura T, Okamoto M, Nakakita S, Suzuki A, Saito M, Tamaki R, et al. Antigenic and receptor binding properties of enterovirus 68. J Virol. 2014;88(5):2374-84. https://doi. org/10.1128/JVI.03070-13 PMID: 24371050

29. Zhang Y, Moore DD, Nix WA, Oberste MS, Weldon WC. Neutralization of Enterovirus D68 isolated from the 2014 US outbreak by commercial intravenous immune globulin products. J Clin Virol. 2015;69:172-5. https://doi.org/10.1016/j. jCv.2015.06.086 PMID: 26209401

30. Pons-Salort M, Grassly NC. Serotype-specific immunity explains the incidence of diseases caused by human enteroviruses. Science. 2018;361(6404):800-3. https://doi. org/10.1126/science.aat6777 PMID: 30139872

\section{License, supplementary material and copyright}

This is an open-access article distributed under the terms of the Creative Commons Attribution (CC BY 4.0) Licence. You may share and adapt the material, but must give appropriate credit to the source, provide a link to the licence and indicate if changes were made.

Any supplementary material referenced in the article can be found in the online version.

This article is copyright of the authors or their affiliated institutions, 2019. 